\title{
Estudo etnofarmacognóstico da saracuramirá (Ampelozizyphus amazonicus Ducke), uma planta medicinal usada por comunidades quilombolas do Município de Oriximiná-PA, Brasil
}

\author{
Danilo Ribeiro de OLIVEIRA ${ }^{1}$, André Luiz Maiocchi Alves COSTA², Gilda Guimarães LEITÃO ${ }^{3}$, Newton \\ Gonçalves CASTRO ${ }^{4}$, Joaquim Pereira dos SANTOS ${ }^{5}$, Suzana Guimarães LEITÃO ${ }^{6}$
}

\begin{abstract}
RESUMO
Um levantamento etnobotânico realizado em comunidades quilombolas de Oriximiná, Pará, destacou a saracuramirá (SAR), Ampelozizyphus amazonicus Ducke, com vasto uso popular no tratamento da malária, como tônica e depurativa. Por este motivo, o presente trabalho objetivou realizar um estudo etnofarmacognóstico da SAR nas respectivas comunidades. Por meio de uma análise etnobotânica quantitativa, foi verificado que SAR apresentou-se dentre as 10 espécies mais versáteis pela elevada importância relativa $(1,3)$, dentre as cinco espécies com maior importância cultural pelo elevado índice de saliência $(0,311)$ e a espécie com maior concordância de uso principal para malária $(85,7 \%)$. Uma análise do índice de espuma e do índice de hemólise para SAR demonstra a presença de saponinas com elevado índice de espuma (833) e uma baixa atividade hemolítica $\left(\mathrm{CH}_{50} 2,6 \mathrm{mg} \mathrm{mL}^{-1}\right)$. Para realizar uma análise das agliconas das saponinas de $\mathrm{SAR}$, a bebida preparada pelo método tradicional quilombola (BMT) foi hidrolisada e, após reação com diazometano, foi analisada por cromatografia gasosa. Dois sinais majoritários foram caracterizados por espectrometria de massas, um referente a um triterpeno de esqueleto damarânico, característico das saponinas da SAR, e outro referente ao éster metílico do ácido betulínico. Partindo das informaçôes de uso popular da SAR, foi avaliada in vitro a atividade inibidora da acetilcolinesterase. Apesar de BMT não ter mostrado atividade neste ensaio, é possível supor que as indicaçóes de uso desta planta pelos quilombolas como fortificante e contra malária podem estar relacionadas a uma possível atividade adaptógena e imunoestimulante, dada à presença das saponinas e do ácido betulínico em BMT.
\end{abstract}

PALAVRAS-CHAVE: Etnofarmacologia, efeito adaptógeno, malária, saponinas, ácido betulínico.

\section{Ethnopharmacology Study of Saracuramirá (Ampelozizyphus amazonicus Ducke) in the "Quilombola" communities of Oriximiná, Pará State, Brazil}

\begin{abstract}
In an ethnobotanical survey conducted within "Quilombola" communities of Oriximiná, Pará State, "saracuramirá" (SAR), Ampelozizyphus amazonicus Ducke, stood out as one of the most cited species with wide popular use, especially for malaria, as tonic and depurative. The aim of this paper was to carry out an ethnopharmacognostic study of SAR in these communities. Quantitative ethnobotanical analysis of the data showed that SAR stands out as one of the 10 most versatile species with a high relative importance $(1,3)$; it remains among the five species with the greatest cultural importance, demonstrated by the high salience index $(0,311)$, being the species with the highest major use agreement to malaria $(85,7 \%)$. Analysis of the foam and hemolysis indexes of SAR shows the presence of saponins with high foam index (833) and low hemolytic activity $\left(\mathrm{HD}_{50} 2,6\right.$ $\mathrm{mg} \mathrm{mL}^{-1}$ ). In order to analyze SAR saponin aglycones, the drink was prepared by the traditional quilombola method (BMT), was hydrolyzed, and analyzed by gas chromatography after reaction with diazomethane. Two major peaks were characterized by mass spectrometry, one referring to a dammarane triterpene skeleton, characteristic from the SAR saponins, and the other identified as the methyl ester of betulinic acid. Owing to the popular usage of SAR, its in vitro acetylcholinesterase inhibitory activity was evaluated, but with negative results. However, it is possible to suggest that the indications of this plant as a tonic and for treating malaria may be related to an adaptogen and immunostimulant effect due to the presence of saponins and betulinic acid in BMT.
\end{abstract}

KEYWORDS: Ethnopharmacology, adaptogen effect, malaria, saponins, betulinic acid

\footnotetext{
1 Universidade Federal do Rio de Janeiro. oliveiradr@pharma.ufrj.br

2 Universidade Federal do Rio de Janeiro. deckioc@uol.com.br

3 Universidade Federal do Rio de Janeiro. ggleitao@nppn.ufrj.br

${ }^{4}$ Universidade Federal do Rio de Janeiro. ngcastro@farmaco.ufrj.br

${ }^{5}$ Associação de Comunidades Remanescentes de Quilombo do Município de Oriximiná. arqmo@hotmail.com

${ }^{6}$ Universidade Federal do Rio de Janeiro. sgleitao@pharma.ufrj.br
} 


\section{INTRODUÇÃO}

Estima-se que em todo o país existam mais de 3.000 comunidades quilombolas. No Estado do Pará já se sabe da existência de pelo menos 240 , sendo que foi no município de Oriximiná-PA, que pela primeira vez uma comunidade quilombola recebeu o título coletivo de suas terras, no ano de 1995 (Oliveira et al. 2010).

Os remanescentes de quilombo de Oriximiná tiveram suas origens no movimento de fuga e resistência dos negros escravos na regiáo do baixo Amazonas, no final do século XVIII e início do século XIX. Os escravos fugidos, na subida pelo rio Trombetas, optavam por duas rotas: uma rumo aos rios Cuminã e Erepecuru e outra em direção ao Alto Trombetas, para trechos bem acima das cachoeiras, descobrindo barreiras naturais para impedir o trânsito das tropas (Andrade 1995; Acevedo e Castro 1998). Com o fim da escravidão no Brasil, os negros localizados acima do Alto Erepecuru e do Alto Trombetas começaram a descer as cachoeiras e a constituir as comunidades que hoje lá existem. Tais comunidades possuem um relativo isolamento, vivendo em áreas de grande biodiversidade e distantes da área urbana.

Em 2001 foi publicada a Medida Provisória 2.186 dispondo sobre autorização de acesso ao conhecimento tradicional associado (CTA) e ao patrimônio genético no Brasil. O projeto de "Bioprospecçẫo de Plantas Medicinais Utilizadas por Comunidades Quilombolas de Oriximiná PA", desenvolvido junto às comunidades quilombolas desta localidade, obteve, em 2007, a primeira autorização no país para acesso ao CTA para fins de bioprospecção (Oliveira et al. 2010). Como resultado desse projeto foram identificadas 227 espécies vegetais, dentre as 254 plantas empregadas medicinalmente pelos quilombolas, merecendo destaque a "saracuramirá", pela sua grande importância nas comunidades, sendo citada por 44\% dos entrevistados (Oliveira 2009).

O espécime coletado como saracuramirá (SAR) foi identificado como Ampelozizyphus amazonicus Ducke. Segundo Lima (2006), a espécie possui distribuição endêmica na América do Sul, ao longo dos territórios da Amazônia brasileira, venezuelana, colombiana e peruana, estendendo-se até o Equador. Sua ocorrência no Brasil limita-se aos estados do Amazonas, Pará e Roraima, sendo encontrada em florestas de terra firme (Lima 2006), preferencialmente, nas proximidades de igarapés e cachoeiras.

Das cascas e raízes de SAR pode ser preparada uma bebida aquosa com espuma abundante e de sabor amargo, semelhante à cerveja, o que lhe confere os nomes de "cervejinha", "cervejeira", "cerveja-do-mato" e "cerveja-de-índio" (Rodrigues 1989; Vieira 1992; Ducke e Martinez 1994; Revilla 2002; Krettli e Andrade-Neto 2004; Santos et al. 2005). Essas propriedades estão relacionadas ao elevado teor de saponinas na espécie. Um estudo recente demonstrou a presença de 48,4\% de saponinas em um extrato aquoso das raízes de SAR (Silva et al. 2009), sendo que algumas delas já foram isoladas e descritas na literatura, como: 3-O-[ $\beta$-D-glicopiranosil $(1 \rightarrow 2) \alpha$-Larabinopiranosil]-20-O- $\alpha$-L-ramnopiranosil-jujubogenina (Brandão et al. 1992), e ampelozigenina-15 $\alpha$-O-acetil-3-O$\alpha$-L-ramnopiranosil-( $1 \rightarrow 2)-\beta$-D-glicopiranosídeo (Brandão et al. 1993). Além destas saponinas, foram descritas outras substâncias, como os triterpenos: ácido melalêucico, ácido

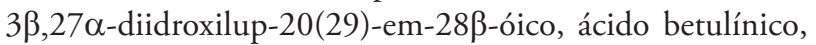
betulina, o lupeol (Brandão et al. 1992); e fitoesteróides (Rosas et al. 2007).

Em toda regiáo amazônica a SAR é tida como uma espécie de extrema importância na prevenção (Brandáo et al. 1985; Vieira 1992; Krettli et al. 2001; Krettli e Andrade-Neto 2004; Silva et al. 2009) e no tratamento de malária (Rodrigues 1989; Ducke e Martinez 1994; Santos e Muaze 2002; Oliveira 2004; Rodrigues 2006). A atividade profilática da SAR já foi demonstrada, in vivo e in vitro, contra a forma esporozoíta de contágio da malária (Andrade Neto et al. 2008), comprovando esta indicação popular da região amazônica.

Considerada uma planta estimulante e energética (Santos et al. 2005), a SAR apresenta ainda outras propriedades importantes descritas na literatura. Suas raízes são tidas como depurativas (Rodrigues 1989; Vieira 1992; Almeida 1993), sendo também empregadas no tratamento de distúrbios gastrintestinais, inflamações e quadros de febre (Rodrigues 2006). O pó das folhas de SAR é indicado como cicatrizante (Almeida 1993; Berg 1993). Suas cascas são utilizadas no preparo de um tônico fortificante (MEB 1993) e afrodisíaco (Berg 1993; Santos et al. 2005), e em casos de mordidas de cobras (Brandáo et al. 1992), no tratamento de gastrite, dores nas juntas, "inflamaçôes de mulher", anemia e diabetes, como revitalizador, depurativo e diurético (Oliveira 2004). Recentemente, foi comprovado em um estudo in vivo o efeito diurético da SAR em ratos (Diniz et al. 2009).

Com o estudo etnobotânico realizado no presente trabalho mostra-se a importância da SAR para as comunidades quilombolas de Oriximiná na prevenção da malária e na promoção da saúde, por meio de métodos etnobotânicos quantitativos. Adicionalmente, procurou-se elucidar, por meio de estudos químicos e farmacológicos, as propriedades atribuídas à bebida preparada com $A$. amazonicus pelo método tradicional utilizado nas respectivas comunidades.

\section{MATERIAL E MÉTODOS}

\section{Caracterização do local de trabalho:}

O município de Oriximiná localiza-se no Oeste do Pará, às margens do rio Trombetas. Limita-se com os municípios de Terra Santa, Faro, Juruti e Óbidos, com o Estado de 
Roraima, com a Guiana e o Suriname. O município possui 33 comunidades quilombolas, distribuídas em oito regiôes distintas: Boa Vista, Água Fria, Trombetas, Erepecuru, Alto Trombetas, Ariramba, Jamari/Último Quilombo e Moura. O trabalho foi realizado nas comunidades Pancada, Jauari e Serrinha, localizadas na área Erepecuru, e nas comunidades de Arancuã-do-Meio e Bacabal, na área Trombetas.

\section{Levantamento etnobotânico}

O presente trabalho recebeu a autorização para acesso ao CTA com fins de bioprospecçáo pelo Conselho de Gestão do Patrimônio Genético (CGEN), por meio da Deliberação n ${ }^{\circ}$ 213 do dia 06 de dezembro de 2007, publicada em Diário Oficial da União dia 27 de dezembro de 2007 (Oliveira et al. 2010).

Para aquisição dos dados etnobotânicos realizaram-se entrevistas, utilizando formulários semiestruturados, para levantamento das espécies medicinais empregadas por 34 quilombolas das cinco comunidades estudadas, sendo esses informantes-chave e especialistas locais, tais como: mateiros, curadores, rezadeiras, benzedeiras, parteiras e puxadeiras ${ }^{1}$. Para análise dos dados etnobotânicos foram utilizados os métodos de listagem-livre para determinação do Índice de Saliência, Concordância de Uso Principal e Importância Relativa.

Listagem Livre e Índice de Saliência (IS) - Foram aplicados formulários para listagem-livre das espécies vegetais utilizadas, sendo o primeiro um formulário simples para a listagem direta das plantas medicinais; e um segundo formulário para abordagem etnodirigida, com perguntas direcionadas à seleçấo e bioprospecçáo de plantas usadas para doenças infecciosas e doença de Alzheimer. Em um contexto coletivo esta abordagem sugere elementos culturalmente mais importantes e em uma ordem de importância (Albuquerque e Lucena 2004). Para tal, foi calculado o Índice de Saliência (IS) com auxílio do programa ANTHROPAC 4.0 (Analytic Technologies, USA).

Concordância de Uso Principal (CUP) - Foi calculada por meio da razão entre o número de entrevistados que citaram de forma independente os usos principais $\left(\mathrm{UP}_{\mathrm{SAR}}\right)$ da SAR, e o número total de entrevistados que mencionaram a SAR para qualquer uso (uso total, $\mathrm{TE}_{\mathrm{SAR}}$ ), conforme a equação: $C U P=U P \times 100 / T E($ Oliveira et al. 2006). Um fator de correção (FC) foi aplicado para calcular a CUP corrigida (CUPc), através da fórmula $C U P c=C U P \times F C$. FC, que é a razão entre o número de entrevistados que citaram a SAR $\left(\mathrm{TE}_{\mathrm{SAR}}\right)$ e o número de entrevistados que citaram a espécie mais citada $\left(\mathrm{TE}^{+}\right)$, no caso o cumaru $(\mathrm{CUM}=$ Dypterix odorata (Aubl.) Willd.= 24 informantes), sendo empregada, portanto, a equação $\mathrm{FC}=\mathrm{TE}_{\mathrm{SAR}} / \mathrm{TE}^{+}{ }_{\mathrm{CUM}}$.

${ }^{1}$ Pessoa que se dedica ao tratamento de problemas do sistema músculo-esquelético, semelhante ao quiroprático.
Importância Relativa (IR) - Estabelece que uma planta seja mais importante quanto mais versátil ela for, ou seja, quanto maior o número de propriedades distintas ela apresentar. $\mathrm{O}$ valor máximo que uma espécie pode obter é dois (Albuquerque e Lucena 2004). O cálculo é feito de acordo com a fórmula $I R=N S C+N P$, em que, NSC = número de sistemas corporais e NP = número de propriedades. NCS é dado pelo número de sistemas corporais tratados por uma determinada espécie (NSCE) dividido pelo número total de sistemas corporais tratados pela espécie mais versátil (NSCEV), sendo esta a castanheira (CAS $=$ Bertholletia excelsa Kunth., $\left.\mathrm{IR}_{\mathrm{CAS}}=2,00\right)$, com a fórmula $N S C=N S C E / N S C E V_{C A S}$. NP é o número de propriedades atribuídas à determinada espécie (NPE) dividido pelo número total de propriedades atribuídas à espécie mais versátil $\left(\mathrm{NPEV}_{\mathrm{CAS}}\right)$, conforme a fórmula $N P=N P E / N P E V$. O cálculo deste índice foi baseado na distribuição das indicaçôes de uso das etnoespécies em sistemas corporais, relacionados a 17 categorias da Classificação Estatística Internacional de Doenças e Problemas Relacionados à Saúde - CID-10 (OMS 1998).

\section{Coleta do material e preparo dos extratos da SAR}

SAR foi coletada nas comunidades Pancada e Bacabal no município de Oriximiná, sendo o material depositado no Herbário do Instituto Nacional de Pesquisas da Amazônia (INPA n ${ }^{\circ}$. 224161).

Para o preparo dos extratos, inicialmente, foi realizada a mondagem das amostras de SAR coletadas, a retirada e a secagem das cascas do cipó. Em seguida, as extraçôes foram obtidas por dois métodos: um por Maceração Simples (MS), em que as cascas da SAR foram agitadas com água por cerca de cinco minutos e deixadas em repouso por 24 horas; e outro pelo Método Tradicional (BMT) de preparo, conforme a técnica das comunidades quilombolas, no qual as cascas da SAR foram agitadas com água até formar espuma abundante e persistente, que foi retirada com o auxílio de uma escumadeira e, entâo, desprezada. Repetiu-se esse procedimento por seis vezes para eliminaçóes sucessivas da espuma. Ambos os extratos foram preparados na proporção de $1 \%(\mathrm{p} / \mathrm{v})$ em água destilada, sendo posteriormente filtrados e liofilizados. Todos os procedimentos foram realizados em triplicata.

\section{Avaliação semiquantitativa de saponinas da SAR}

Foi realizada a determinaçáo do índice de espuma (IE) para as cascas da planta segundo procedimento descrito no manual de técnicas de controle de qualidade para drogas vegetais, da Organização Mundial da Saúde (WHO 1998). Foi utilizado como padrão as cascas do juazeiro (Ziziphus joazeiro Mart., Rhamnaceae) coletadas no município de Alagoinhas, Bahia, pertencente ao acervo de drogas vegetais da cadeira de Farmacognosia da Faculdade de Farmácia da Universidade Federal do Rio de Janeiro. A escolha do juazeiro como padrão 
foi devida à abundância de saponinas e ao seu elevado índice de espuma, previamente descritos na literatura (Kato 1996).

\section{Hidrólise do extrato da SAR e análise cromatográfica}

Para realizar a hidrólise de BMT, inicialmente, $1 \mathrm{~g}$ do extrato liofilizado (pó) foi dissolvido em $30 \mathrm{~mL}$ de água destilada e foram feitas seis extraçôes com hexano. Após a extração, evaporou-se o restante do solvente orgânico da fase aquosa em evaporador rotatório e adicionou-se etanol à fase aquosa na proporção de 1:1. Nesta solução, foi feita uma precipitação de substâncias fenólicas utilizando-se $10 \mathrm{~mL}$ de soluçáo de acetato de chumbo (10\%) sob refluxo por 15 min. O precipitado formado foi separado por centrifugaçáo, e o sobrenadante foi evaporado para retirada do etanol. A hidrólise foi feita adicionando-se $3 \mathrm{~mL}$ de $\mathrm{HCl}$ concentrado $(37 \% \mathrm{v} / \mathrm{v})$ e mantendo a solução por $4 \mathrm{~h}$ sob refluxo, sendo observada a formação de cristais brancos em forma de agulha. $\mathrm{O}$ hidrolisado foi posteriormente neutralizado com $\mathrm{KOH}$ até pH 7, e entấo extraído com diclorometano. A fase orgânica foi evaporada e o produto obtido foi metilado com diazometano segundo a literatura (Biavatti et al. 2006).

A análise do hidrolisado por cromatografia gasosa foi realizada por: CG-DIC, em um cromatógrafo Shimadzu, modelo GC-SC 2010 (Japão), coluna ZB-5MS (30 m x 0,25 $\mathrm{mm} \times 0,25 \mu \mathrm{m})$, temperatura de injetor $270^{\circ} \mathrm{C}$, programa de variaçấo da temperatura da coluna de $150^{\circ} \mathrm{C}-270^{\circ} \mathrm{C}(5$ $\left.{ }^{\circ} \mathrm{C} \mathrm{min}^{-1}\right)$, sendo utilizado o Hidrogênio como gás de arraste; CG-EM: cromatógrafo Shimadzu, modelo GCMS-QP 2010 Plus (Japão), em coluna ZB-5MS (30 m x 0,25 mm x 0,25 $\mu \mathrm{m})$, temperatura de injetor $270^{\circ} \mathrm{C}$, temperatura de interface $230^{\circ} \mathrm{C}$, e programa de variaçáo da temperatura da coluna de $150^{\circ} \mathrm{C}-270^{\circ} \mathrm{C}\left(5^{\circ} \mathrm{C} \mathrm{min}^{-1}\right)$, sendo utilizado o Hélio como gás de arraste. Os dados foram comparados com os dados da Biblioteca espectral NIST (National Institute of Standards and Technology). $\mathrm{T}_{\mathrm{R}}$ 26,62 min $\mathrm{m} / \mathrm{z}(\%): 350(8,3) ; 348(33,3)$; 333 (8,3); 315 (16,6); 297 (5,5); 274 (33,3); 257 (16,6); 233 (5,5); 207 (8,3); 189 (27,7); 175 (16,6); 161 (16,6); 147 (22,2); 135 (100); 121 (69,4); 109 (69,4); 95 (77,2); 81 $(58,3) ; 69(55,5) ; 43(72,2) . \mathrm{T}_{\mathrm{R}} 37,44 \mathrm{~min} \mathrm{~m} / \mathrm{z}(\%): 470(8,3)$; $452(5,5) ; 437(2,7) ; 411(11,1) ; 395(2,7) ; 377(1,1) ; 356$ $(0,5) ; 343(0,5) ; 330(1,1) ; 316(1,6) ; 301(1,1) ; 284(0,5)$; 273 (5,5); 262 (47,2); 248 (16,6); 233 (16,6); $220(30,5)$; 203 (47,2); 189 (100); 175 (38,8); $161(16,6) ; 147(27,7)$; 133 (38,8); 119 (55,5); $107(50) ; 95(58,3) ; 81(52,7) ; 69$ $(47,2) ; 55(41,6)$.

\section{Atividade inibidora da acetilcolinesterase da SAR}

A atividade da acetilcolinesterase (AChE) obtida de peixeelétrico (Electrophorus electricus) foi medida de acordo com o método de Ellman (Ellman 1961) modificado por Oliveira et al. (2007) usando o espectrofotômetro SpectraMax 250 (Molecular Devices Corp., USA) com microplaca de 96 poços, sendo adicionados aos poços $15 \mu \mathrm{L}$ de AchE (tipo VI-S, C3389, Sigma-Aldrich, St. Louis, USA) a 0,23U mL $\mathrm{mL}^{-1}$ (em $50 \mathrm{mM}$ de tampáo Tris- $\mathrm{HCl}, \mathrm{pH} 8$, e $0,1 \%$ de albumina de soro bovino), $10 \mu \mathrm{L}$ de ácido 5,5'-ditio-bis-(2-nitrobenzóico (D8130, Sigma-Aldrich, St. Louis, USA) a $5 \mathrm{mM}$ (em $50 \mathrm{mM}$ de tampáo Tris- $\mathrm{HCl}$, pH 7 e $80 \mathrm{mM}$ de $\left.\mathrm{MgCl} .6 \mathrm{H}_{2} \mathrm{O}\right), 20 \mu \mathrm{L}$ das soluçôes de BMT $5 \mathrm{mg} \mathrm{mL}^{-1}$ (em tampáo Tris- $\mathrm{HCl}, \mathrm{pH}$ 8), $145 \mu \mathrm{L}$ do tampáo Tris- $\mathrm{HCl}, \mathrm{pH} 8$ e $0,1 \%$ de albumina de soro bovino e $10 \mu \mathrm{L}$ de iodeto de acetiltiocolina (A 5751, Sigma-Aldrich, St. Louis, USA) a $10 \mathrm{mM}$ em água destilada. Nas placas foram adicionados os controles para atividade da AChE (mais $20 \mu \mathrm{L}$ de tampão substituindo a amostra), controle da inibição do solvente e o controle positivo, a fisostigmina (Fluka, No 45710, Buchs, Switzerland). O aumento da absorvância foi medido em $412 \mathrm{~nm}$ durante 5 min, a cada $13 \mathrm{~s}$. A velocidade de reaçấo foi calculada pelo programa Soft Max Pro 4.0, USA. Os experimentos foram realizados em triplicata.

\section{Atividade hemolítica da SAR}

O ensaio foi realizado segundo o método de Yang et al. (2005) modificado. Para o ensaio foram obtidas hemácias de voluntário saudável, coletando-se o sangue com o sistema a vácuo Vacuette ce ${ }^{\circledR}$ (K3EDTA; Greiner BIO-ONE). Uma alíquota de $4 \mathrm{~mL}$ do sangue foi lavada três vezes com soluçáo salina $(0,9 \% \mathrm{p} / \mathrm{v}, \mathrm{NaCl})$ por centrifugação a 3000 $\mathrm{rpm}$, durante $5 \mathrm{~min}$, sendo o sobrenadante descartado. Os eritrócitos sedimentados no tubo foram diluídos em solução salina até obtenção de uma suspensão a $1 \%(\mathrm{v} / \mathrm{v})$. Um volume de $0,5 \mathrm{~mL}$ desta suspensão de células foi misturado a 0,5 $\mathrm{mL}$ de diluentes contendo concentraçôes de 250 a 4500 $\mu \mathrm{g} \mathrm{mL} L^{-1}$ de BMT. As misturas foram incubadas a $37^{\circ} \mathrm{C}$, com agitação contínua, durante $60 \mathrm{~min}$. As soluçôes foram, então, centrifugadas a $3.000 \mathrm{rpm}$ por $5 \mathrm{~min}$. A absorvância do sobrenadante foi medida a $540 \mathrm{~nm}$ no espectrofotômetro SpectraMax 250 (Molecular Devices Corp., EUA). As suspensões de hemácias acrescidas de solução salina e de água destilada foram, respectivamente, os controles hemolíticos mínimo e máximo. Todos os experimentos foram realizados em triplicata. A concentração hemolítica média $\left(\mathrm{CH}_{50}\right)$ foi estimada por regressão não linear com o software GraphPad Prism 4 (EUA). Saponina Merck ${ }^{\circledR}$ foi utilizada como controle positivo nas concentraçôes de 5 a $1.000 \mu \mathrm{g} \mathrm{mL}^{-1}$ Para eliminar a interferência dos extratos na absorvância, foram preparadas soluçôes controle (branco), nấo havendo a adição da solução de hemácias.

\section{RESULTADOS E DISCUSSÃO}

\section{Estudo etnobotânico}

No levantamento etnobotânico realizado entre 2006 e 2008, foram citadas um total de 254 plantas pelos 34 
informantes entrevistados, destacando-se a saracuramirá (SAR) por sua frequência de uso, tendo sido citada por 15 deles. Ela se destaca, ainda, por estar entre as cinco espécies com maior CUPc para as comunidades quilombolas pesquisadas (Oliveira 2009). Os principais usos terapêuticos citados para as cascas de SAR foram: contra malária $(\mathrm{CUP}=85,7 \%$; CUPc $=$ $50,0 \%)$, doenças do fígado (CUP $=35,7 \%$, CUPc $=20,8 \%$ ), e como tônico para os nervos e depurativo do sangue (CUP = $14,3 \%$; CUPc $=8,3 \%)$. Outros usos secundários, com valores de CUP menores do que 10\%, foram: memória, anemia, dores estomacais, indisposição, inflamação de próstata, falta de apetite, intoxicação, doenças dos rins, diabetes e como afrodisíaco e purgativo. Os usos citados para SAR nas comunidades quilombolas de Oriximiná estão parcialmente em conformidade com alguns usos populares já descritos na literatura, como para o tratamento e prevençáo de malária, para desordens do fígado, como tônica e depurativa (Brandão et al. 1985; Rodrigues 1989; Vieira 1992; Almeida 1993; Berg 1993; MEB 1993; Ducke e Martinez 1994; Krettli et al. 2001; Santos e Muaze 2002; Oliveira 2004; Santos et al. 2005; Rodrigues 2006), já que esta ainda foi indicada para memória, inflamação de próstata, falta de apetite, prisão de ventre e tratamento de intoxicação.
$\mathrm{Na}$ análise da Importância Relativa, a SAR está entre as 10 espécies mais versáteis $(\mathrm{IR}=1,3)$ para as comunidades quilombolas estudadas, tendo um uso diversificado conforme as 15 propriedades de usos citadas (NPE), incluídas em oito sistemas corporais (NSCE) bem distintos (Tabela 1).

A técnica de listagem livre permitiu analisar a importância cultural das espécies medicinais empregadas nas comunidades, estando a SAR entre as cinco espécies com maior saliência $(I S=0,311)$ (Tabela 2). A diversidade de usos demonstrada pelo elevado IR para a SAR, bem como a constataçáo secular dos benefícios de seu uso terapêutico fazem com que a SAR seja uma espécie de primeira escolha no tratamento de diversos males, sendo sempre lembrada rapidamente entre os entrevistados.

\section{Avaliação etnofarmacognóstica de SAR}

As comunidades quilombolas de Oriximiná utilizam medicinalmente apenas as cascas da SAR, o que torna o manejo da espécie mais fácil, diferente de outras comunidades amazônicas que fazem o emprego das raízes (Krettli et al. 2001). Além disso, o método utilizado para preparar a SAR é frequentemente diferente da técnica de infusão, muito citada na literatura (Santos e Muaze 2002; Oliveira 2004)

Tabela 1 - Propriedades de uso citadas pelos quilombolas de Oriximiná para a saracuramirá, segundo a Classificação Estatística Internacional de Doenças e Problemas relacionados à Saúde - CID-10 (OMS 1998).

\begin{tabular}{lc}
\hline $\begin{array}{l}\text { No de Sistemas Corporais Tratados por uma Determinada Espécie (NSCE), } \\
\text { conforme a CID-10 }\end{array}$ & No de propriedades atribuídas à determinada espécie (NPE) \\
\hline I - Algumas Doenças Infecciosas e Parasitárias & Malária \\
III - Doenças do Sangue, Órgãos Hematopoiéticos e Transtornos Imunitários & Anemia, depurativo do sangue \\
IV - Doenças Endócrinas, Nutricionais, Metabólicas & Diabetes \\
VI - Doenças do Sistema Nervoso & Tônico para os nervos, memória, afrodisíaco, falta de apetite \\
XI - Doenças do Aparelho Digestivo & Doenças do fígado, dores estomacais e purgativo \\
XIV - Doenças do Aparelho Gênito-Urinário & Inflamação de próstata, rins \\
XVIII - Sintomas, Sinais e Achados Anormais (Afecções Maldefinidas) & Indisposição \\
XIX - Lesões, Envenenamentos e algumas Consequências de Causas Externas & Intoxicação \\
\hline
\end{tabular}

Tabela 2 - Índice de Saliência (IS) obtido para as espécies citadas na listagem-livre (indicações gerais) e na listagem-livre etnodirigida para malária e doenças relacionadas e para distúrbios da memória e desordens relacionadas:

\begin{tabular}{|c|c|c|c|c|c|}
\hline $\begin{array}{l}\text { Espécies mais salientes } \\
\text { "indicações gerais" }\end{array}$ & IS & $\begin{array}{l}\text { Espécies mais salientes } \\
\text { "para malária" }\end{array}$ & IS & $\begin{array}{l}\text { Espécies mais salientes } \\
\text { "para memória" }\end{array}$ & IS \\
\hline $\begin{array}{c}\text { 1- Arruda } \\
\text { Ruta graveolens L. }\end{array}$ & 0,372 & $\begin{array}{c}\text { 1- Saracuramirá } \\
\text { Ampelozizyphus amazonicus Ducke }\end{array}$ & 0,360 & $\begin{array}{c}\text { 1- Jutaí/Jatobá } \\
\text { Hymenaea intermedia Ducke }\end{array}$ & 0,253 \\
\hline $\begin{array}{l}\text { 2- Cumaru } \\
\text { Dipteryx odorata (Aubl.) Willd }\end{array}$ & 0,365 & $\begin{array}{c}\text { 2- Carapanaúba } \\
\text { Aspidosperma rigidum Rusby }\end{array}$ & 0,251 & $\begin{array}{c}\text { 2- Cumaru } \\
\text { Dipteryx odorata (Aubl.) Willd }\end{array}$ & 0,201 \\
\hline $\begin{array}{l}\text { 3- Peão-branco } \\
\text { Jatropha curcas L. }\end{array}$ & 0,366 & $\begin{array}{l}\text { 3- Quinarana } \\
\text { Geissospermum argentum Woodson }\end{array}$ & 0,249 & $\begin{array}{l}\text { 3- Coco-verde } \\
\text { Cocos nucifera L. }\end{array}$ & 0,194 \\
\hline $\begin{array}{c}\text { 4- Mastruz } \\
\text { Chenopodium ambrosioides L. }\end{array}$ & 0,33 & $\begin{array}{l}\text { 4- Pau-paratudo } \\
\text { Simaba cedron Planch }\end{array}$ & 0,148 & $\begin{array}{c}\text { 4- Marapuama } \\
\text { Ptychopetalum olacoides Benth }\end{array}$ & 0,174 \\
\hline $\begin{array}{c}\text { 5- Saracuramirá } \\
\text { Ampelozizyphus amazonicus Ducke }\end{array}$ & 0,311 & $\begin{array}{c}\text { 5- Castanheira } \\
\text { Bertholletia excelsa H.B.K. }\end{array}$ & 0,124 & $\begin{array}{c}\text { 5- Saracuramirá Ampelozizyphus } \\
\text { amazonicus Ducke }\end{array}$ & 0,113 \\
\hline
\end{tabular}


e semelhante ao método descrito por Rodrigues (1989), em que o caule é levemente raspado, sendo as cascas batidas numa vasilha com água. No método quilombola, no entanto, a espuma formada com a agitação das cascas na água deve ser desprezada por sete vezes, durante pelo menos 10 minutos de agitação vigorosa. Quando os comunitários eram arguidos sobre porque a SAR preparada nas comunidades quilombolas era "batida" sete vezes na água, muitos relatavam que se não fosse retirada a espuma, a bebida poderia "fazer mal, ou dar tontura e enjoo", enquanto alguns afirmavam estar apenas relacionado ao processo de extração. Alguns quilombolas também fazem o chá do lenho (sem as cascas) do cipó da SAR e das folhas, e os consomem alegando que estes também possuem os mesmos efeitos, porém mais fracos que as cascas. Com este relato demonstra-se um potencial para o uso de outros órgãos vegetais da SAR, o que contribuiria no melhor aproveitamento e na conservação da espécie, mesmo se a espécie vier a ser cultivada e domesticada.

Ao analisar o teor dos extrativos obtidos, observou-se uma diferença entre os métodos de extração por maceração simples (MS) e o método tradicional (BMT) das comunidades. Para o primeiro, obteve-se um rendimento de $11,2 \pm 0,33 \%$ de pó liofilizado, enquanto que para o segundo obteve-se rendimento de 9,6 $\pm 0,19 \%$, demonstrando uma diferença significativa entre os métodos pelo teste $t(\mathrm{t}=7,2759)$. A agitação vigorosa promovida para a formaçáo e retirada da espuma promove uma redução do teor de extrativos obtidos, possivelmente saponinas, já que a parte desprezada é a espuma. Apesar da pequena diferença com relação ao teor de extrativos quando se compararam ambos os métodos, a afirmaçáo dos quilombolas de que o modo de preparo tem o objetivo de evitar enjoo pode estar relacionado ao fato de que drogas com alto teor de saponinas frequentemente promovem irritação gástrica quando ingeridas por via oral.

A propriedade detergente da $S A R$ já é relatada na literatura (Vieira 1992; Almeida 1993), no entanto, não havia sido descrito um índice de espuma (IE) para a espécie até o momento. Visto que este é uma determinação farmacopéica importante para plantas com saponinas, realizou-se uma avaliação semiquantitativa do teor de saponinas das cascas da planta IE, obtendo-se um resultado bem elevado para o índice de espuma, com IE $=833$. O resultado obtido foi semelhante ao do controle positivo para as cascas de $Z$. joazeiro $(\mathrm{IE}=800)$. Esta espécie, que também pertence à família Rhamnaceae, é caracterizada pelo alto teor de saponinas, sendo amplamente empregada em produtos para a escovação e higiene bucal.

Com o objetivo de avaliar a natureza das agliconas que compóem as saponinas presentes na bebida, foi realizada a hidrólise de BMT. Após o procedimento de hidrólise foi obtido um sólido com $5 \%$ de rendimento $(\mathrm{p} / \mathrm{p})$, que foi analisado por CG-DIC e CG-EM. O cromatograma obtido (CG-DIC) apresentou um total de 48 sinais, sendo dois majoritários: $T_{R}=$ $26,62 \min (38 \%) \mathrm{e} \mathrm{T}_{\mathrm{R}}=37,44 \mathrm{~min}(21 \%)$ (Figura 1). O sinal em $T_{R}=26,62$ min apresentou um fragmentograma no CGEM compatível com um triterpeno de esqueleto damarânico que não pode ser identificado, porém, é consistente com as estruturas dos constituintes químicos identificados na espécie (Brandáo et al. 1992; 1993). O sinal em $\mathrm{T}_{\mathrm{R}}=37,44$ min foi identificado como o éster metílico do ácido betulínico. Até o presente momento, as únicas saponinas isoladas e descritas para SAR são do tipo damarânicas. A presença do éster metílico do ácido betulínico no hidrolisado de BMT permite supor que o mesmo é extraído durante o processo de preparação de BMT, que não envolve calor. $\mathrm{O}$ ácido betulínico é um triterpeno insolúvel em água, porém a presença de saponinas em BMT pode ter atuado como um agente modificador desta solubilidade. Dessa forma, foi investigada a presença do ácido betulínico livre em BMT, por meio de cromatografia em camada fina, com padrão do mesmo, o que confirmou a presença do ácido livre em BMT (resultados não mostrados).

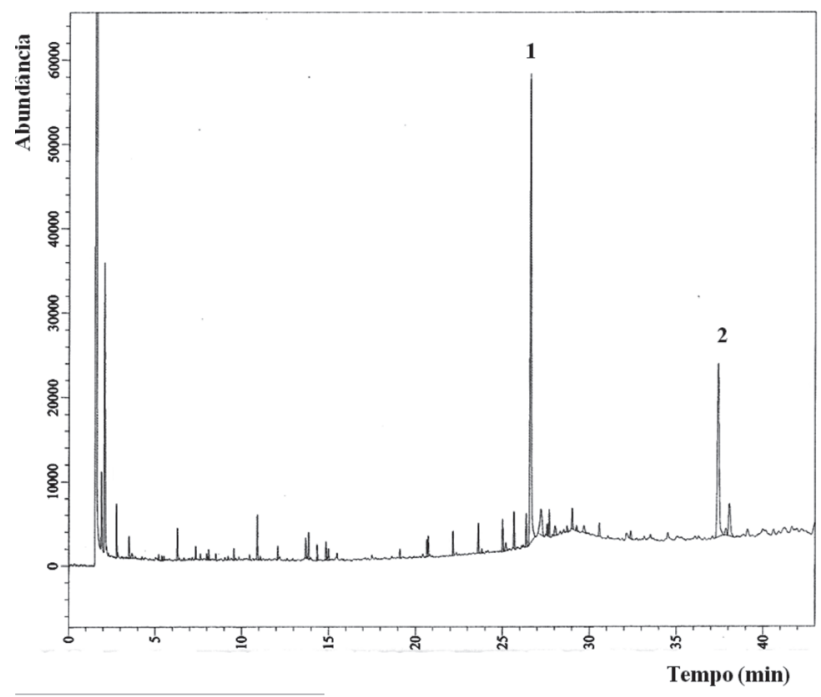

Figura 1 - Cromatograma CG-DIC do produto de hidrólise de MT, após reação com diazometano. Sinal $1\left(T_{R}=26,62 \mathrm{~min}\right.$ ) referente a um triterpeno de esqueleto damarânico e Sinal $2\left(T_{R}=37,44\right.$ min) éster metílico do ácido betulínico.

\section{Avaliação das propriedades biológicas de SAR}

Quando aplicou-se a técnica da listagem livre associada a uma abordagem etnodirigida para o tratamento de malária e de sintomas associados, a SAR torna-se a espécie com maior saliência (IS =0,36) (Tabela 2), sendo, dentre as 35 etnoespécies utilizadas nas comunidades para esse fim, a mais importante (Oliveira 2009). A lista livre pode identificar itens dentro de uma categoria êmica, ou um domínio cultural, e pode acumular dados diretos de maneira fácil e simples, podendo ser utilizada, inclusive, como uma técnica exploratória (Thompson e Juan 2006). 
Embora a SAR seja a etnoespécie com maior saliência para malária $(\mathrm{IS}=0,36)$ e frequência de citaçóes $(\mathrm{FR}=50 \%)$, elevada importância relativa $(\mathrm{IR}=1,1)$ e concordância de uso principal $(\mathrm{CUP}=100 \%$ e CUPc $=50 \%)$ deste estudo, uma análise em colaboração com a Dra. Antoniana U. Krettli (dados não mostrados) revelou que suas cascas náo apresentam atividade antiplasmódica in vitro contra os parasitas sanguíneos do Plasmodium falciparum.

Outros estudos já haviam descrito o mesmo resultado negativo para atividade da SAR contra formas sanguíneas do plasmódio (Brandão et al. 1985; Carvalho et al. 1992; Andrade-Neto et al. 2008). A atividade parcial da SAR foi demonstrada contra a forma esporozoíta de Plasmodium gallinaceum in vivo, com a administração do extrato da planta e inoculaçáo dos parasitas em galinha (Krettli et al. 2001). Estudos realizados mais recentemente in vivo e in vitro com esporozoítas de Plasmodium berghei trouxeram evidências diretas da açáo antiesporozoíta da SAR, que confirmam a hipótese desta ser uma planta profilática, apesar de não ser curativa. Foi demonstrado, em culturas in vitro de esporozoítas pré-incubados com extratos de SAR, uma reduçáo do número de células hepáticas infectadas, além de um reduzido número de esquizontes celulares. Camundongos tratados com doses mais elevadas de extratos de SAR e em seguida inoculados com esporozoítas do $P$. berghei náo se tornaram infectados, ou tiveram baixa parasitemia e atrasaram o desenvolvimento da doença (Andrade-Neto et al. 2008). Sendo os esporozoítas a forma de contágio do plasmódio, tal resultado confirma a atividade profilática da SAR contra malária.

$\mathrm{O}$ mecanismo desta atividade profilática ainda não foi esclarecido e necessita ser investigado, entretanto, é possível que essa ação possa ser explicada pela sua composição rica em saponinas. Essas substâncias possuem ação detergente e podem ser capazes de alterar a permeabilidade da membrana dos esporozoítas e prejudicar seus movimentos explicando a baixa infecção. Segundo Andrade-Neto et al. (2008), possivelmente ocorre uma interaçáo entre as saponinas da planta com os esporozoítas antes de chegarem à forma hepática inibindo a sua posterior evoluçáo no fígado.

Até o momento só existem evidências científicas para efeito profilático da SAR. O seu extenso uso como antimalárico curativo náo foi confirmado em estudos in vitro e in vivo com $P$. falciparum e $P$. berghei. Nas comunidades quilombolas dizem que a "cervejinha" previne, cura e impede o retorno da doença. A SAR é amplamente empregada pelos quilombolas como depurativa ajudando a "eliminar aquilo que não presta do organismo", "limpando o sangue e o fígado".

${ }^{2}$ Diz-se do alimento que tem reima, isto é, que produz prurido (urticária) ou se exterioriza por piora de manifestações cutâneas pré-existentes ou, de modo geral, vai acentuar as "inflamações" do fígado, rins, ovários etc. também pré-existentes (Matos 2007).
Uma possibilidade aqui aventada para a SAR auxiliar na recuperação do paciente com malária seria por uma ação imunoestimulante, conforme seus usos relatados como fortificante, estimulante, para prevenir e combater doenças. Os quilombolas consideram que a doença debilita e a não recuperaçáo da saúde, o uso álcool ou de comida "remosa", como a carne do queixada (Tayassu pecari Link.) ou de jacaré (Caiman spp.), traz a doença de volta, se ela ainda não tiver sido eliminada por completo.

O efeito imunomodulador e imunoestimulante de plantas medicinais é bem descrito na literatura, e em especial, para plantas ricas em saponinas, como Panax ginseng C.A. Meyer, Glycyrrhiza glabra L., Cimicifuga racemosa L., etc. (Francis et al. 2002; Sparg et al. 2004; Patwardham e Gautam 2005). Para o ácido betulínico, cuja presença na bebida preparada pelo método tradicional foi demonstrada nesse estudo, também já foram descritas atividades anticarcinogênica e antiinflamatória. Atualmente, tem sido demonstrado um excelente efeito imunoestimulante para espécies da família Rhamnaceae, por exemplo, para Zyziphus mauritiana Lam., sendo seus extratos ativos padronizados em ácido betulínico (Mishra e Bhatia 2010). Segundo Santos et al. (2005), a utilização da SAR pelos doentes com malária objetiva não a cura, mas o restabelecimento da energia, efeito talvez relacionado à sua composiçáo rica em saponinas, classe com comprovado efeito adaptógeno (Panossian et al. 1999). O termo adaptógeno define substâncias farmacologicamente ativas, capazes de induzir no organismo a um estado de resistência não-específica aumentada, tornando-o capaz de responder melhor ao estresse e adaptar-se ao agente agressor (Panossian et al. 1999). Portanto, o efeito adaptógeno das saponinas da SAR, associada ao ácido betulínico, pode ter um importante papel no sistema imunológico de pacientes infectados pelo Plamodium vivax, impedindo as recaídas da malária.

No levantamento etnodirigido realizado neste estudo por meio da técnica da listagem-livre para as plantas utilizadas nas comunidades quilombolas de Oriximiná com indicaçôes para memória, esquecimento, caduquice, velhice, rejuvenescer, "fraqueza de cabeça", "mente fraca", "fraqueza de homem", "fortificante para os nervos", afrodisíaco, entre outras, foram levantadas 36 etnoespécies; sendo a SAR a planta com o quinto maior Índice de Saliência (IS = 0,113) (Tabela 2).

As indicaçôes da SAR como tônico para os nervos e para memória, como fortificante e afrodisíaco, nos levaram a investigar um possivel efeito inibidor da acetilcolinesterase, já que este é o mecanismo de ação de vários fármacos usados na terapêutica da doença de Alzheimer, que leva o paciente à perda progressiva da memória (Oliveira et al. 2007). Apesar das indicaçốes de uso desta planta para memória, os diferentes extratos da SAR testados não foram capazes de inibir a acetilcolinesterase in vitro, na concentraçâo final de até 0,5 
$\mathrm{mg} \mathrm{mL}^{-1}$, apresentando inibições inferiores a 5\%, comparado com a inibição de 99,9\% da fisostigmina.

A doença de Alzheimer não parece ser comum nas comunidades quilombolas estudadas. A aparente baixa incidência de demências entre quilombolas pode estar relacionada à alimentaçáo, pela frequente ingestão de castanha-do-brasil (B. excelsa) e peixes, muito ricos, respectivamente, em selênio e ácidos graxos poliinsaturados (ex. omega-3). A importância desses elementos na prevenção de doenças neurodegenerativas e na melhora cognitiva de pacientes com Alzheimer já foi demonstrada (Mazza et al. 2007; Scheltens et al. 2010). Além disso, o conceito de memória e de esquecimento nas comunidades está relacionado principalmente a um estado de fraqueza, desnutrição, debilidade causada por doenças diversas, preocupaçóes excessivas etc. A recuperação da memória seria um reflexo da recuperaçáo da saúde como um todo, corroborando com o possível efeito adaptógeno da espécie. Os efeitos benéficos das saponinas sobre a memória e a cognição tem sido bem relatados na literatura (Sparg et al. 2004; Lee et al. 2009).

Segundo relatos dos quilombolas, a SAR usada em abundância faz a pessoa engordar muito e ficar muito forte, tornando-a "macetona", "parrudona" e pode "fazer muito filho" por estar fortalecida. É possível que este efeito esteja relacionado a uma atividade tipo mineralocorticóide (Francis et al. 2002). Alguns quilombolas ainda relatam fazer bem "tomar a SAR pela manhã cedo em jejum, mergulhar no rio e ficar com o corpo quase todo submerso durante alguns minutos, deixando esfriar todo o corpo". O fato de tomar a bebida, preferencialmente, em jejum e antes do banho já havia sido descrito para a infusão das cascas das raízes da SAR para outros ribeirinhos amazônicos (Krettli et al. 2001). Esta prática está relacionada à atividade profilática da bebida, já que de estômago vazio a absorção dos princípios ativos da SAR pode ser mais rápida, além de que a hora do banho é considerada um momento propício para a picada do mosquito Anopheles spp.

Tendo em vista o fato já bastante descrito que algumas saponinas podem apresentar forte poder hemolítico, pela sua capacidade de se ligar às membranas dos eritrócitos causando um aumento de permeabilidade, ruptura das células, com consequente liberação de hemoglobina, resolvemos investigar esta propriedade para as saponinas da SAR. A ação hemolítica pode ser tóxica, principalmente, quando ocorre administração por via intravenosa (Sparg et al. 2004), o que tem representado o maior desafio ao desenvolvimento de medicamentos anticâncer e adjuvantes de vacinas à base de saponinas (Gauthier et al. 2009). Contudo, o extrato de SAR obtido pelo método tradicional (MT) não apresentou nenhuma atividade hemolítica na faixa de concentração entre 250 a $750 \mu \mathrm{g} \mathrm{mL}^{-1}$, e o valor de $\mathrm{CH}_{50}$ foi de apenas $2,6 \mathrm{mg} \mathrm{mL}^{-1}$ (Figura 2a). A atividade hemolítica de MT em eritrócitos humanos foi bem inferior à atividade apresentada pela Saponina $\operatorname{Merck}^{\circledR}\left(\mathrm{CH}_{50}\right.$ $=52 \mu \mathrm{g} \mathrm{mL}^{-1}$ ) (Figura $2 \mathrm{~b}$ ), bem como por extratos de diversas outras espécies vegetais ricas em saponinas em que $\mathrm{CH}_{50}$ pode variar de 23 a $50 \mu \mathrm{g} \mathrm{mL}^{-1}$ (Sparg et al. 2004).

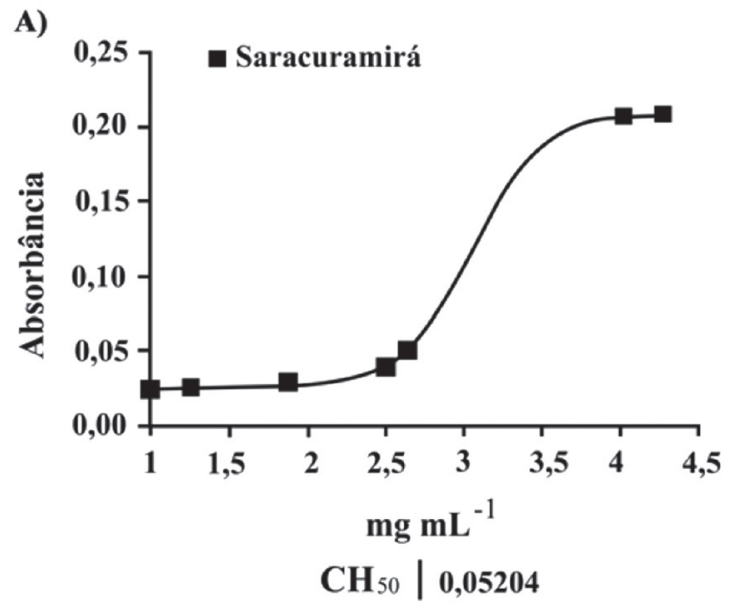

B)

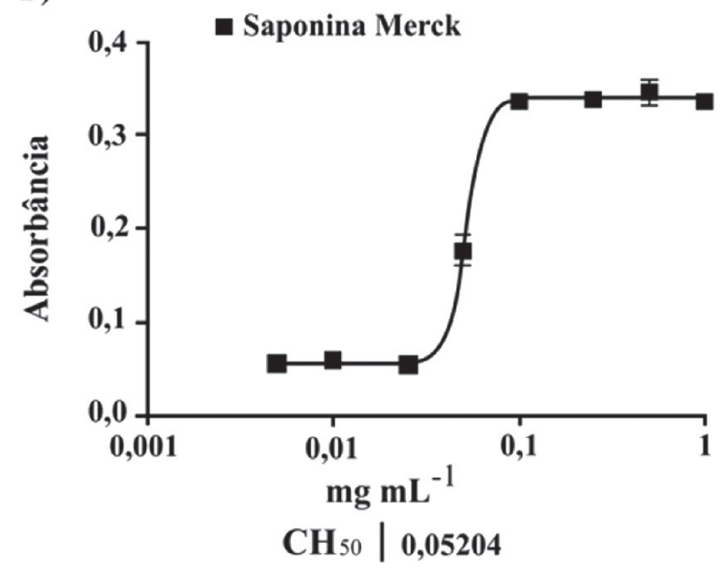

Figura 2 - Curvas Dose-Resposta da atividade hemolítica das amostras (A) Saracuramirá e (B) Saponina Padrão Merck, e seus respectivos valores de $\mathrm{CH}_{50}$

\section{AGRADECIMENTOS}

Os autores agradecem ao Conselho Nacional de Desenvolvimento Científico e Tecnológico (CNPq) pela bolsa de doutorado concedida a Danilo Ribeiro de Oliveira, aos membros das comunidades quilombolas de Oriximiná e à coordenaçáo da Associaçao de Comunidades Remanescentes de Quilombos do Município de Oriximiná. Agradecemos ainda à Profa. Antoniana U. Krettli do Laboratório de Malária do Centro de Pesquisas René Rachou pelos testes para atividade antiplasmódica e pela revisáo crítica do texto, aos mateiros Augusto Figueiredo (Comunidade Pancada) e Celestino Andrade dos Santos (Comunidade Bacabal) pelo auxílio nas coletas de campo, e ao parataxonomista José Ferreira Ramos pela identificaçáo botânica da saracuramirá. 


\section{BIBLIOGRAFIA CITADA}

Acevedo, R.; Castro, E. 1998. Maroons of the Trombetas. Guardians of the forests and rivers. 2Ed. Cejup, Belém, Pará. 262 pp.

Almeida, E.R. 1993. Brazilian medicinal plants. Popular and scientific knowleadge. Editora Helmus, São Paulo, Brasil. 341 pp.

Albuquerque, U.P.; Lucena, R.F.P. 2004. Methods and techniques in the etnobotany research. Livro Rápido/Núcleo de Publicações em Ecologia e Etnobotânica Aplicada, Recife, Pernambuco. 189 pp. (in Portuguese).

Andrade, L.M.M. 1995. Quilombos from the Trombetas river basin: soon historical, p. 47-60. In: O'Dwyer, E.C. (Org.). Lands of Quilombos. Associação Brasileira de Antropologia/ CFCH/UFRJ, Rio de Janeiro, Brasil.(in Portuguese).

Andrade-Neto, V.F.; Brandão, M.G.L.; Nogueira, F.; Rosário, V.E.; Krettli, A.U. 2008. Ampelozyziphus amazonicus Ducke (Rhamnaceae), a medicinal plant used to prevent malaria in the Amazon Region, hampers the development of Plasmodium berghei sporozoites. International Jornal for Parasitology, 38: 1505-1511.

Berg, M.E. 1993. Medicinal plants in the Amazon: contribution to its systematic use. 2 Ed. Rev. Museu paraense Emílio Goeldi, Belém, Pará. 207 pp. (in Portuguese).

Biavatti, M.W.; Dossin, D.; Deschamps, F.C.; Paz Lima, M. 2006. Copaiba oil-resin analysis: contribution to quality control. Revista Brasileira de Farmacognosia, 16: 230-235. (in Portuguese, with abstract in English).

Brandão, M.G.L.; Botelho, M.G.A.; Krettli, A.U. 1985. Experimental antimalarial chemotherapy with natural products: A more rational approach? Ciência e Cultura, 37: 1152-1163. (in Portuguese).

Brandão, M.G.L.; Lacaille-Dubois, M.A.; Teixeira, M.A.; Wagner, H. 1992. Triterpene saponins from the roots of Ampelozizyphus amazonicus. Phytochemistry, 31: 352-354.

Brandão, M.G.L.; Lacaille-Dubois, M.A.; Teixeira, M.A.; Wagner, H. 1993. A Dammarane-type saponin from the roots of Ampelozizyphus amazonicus. Phytochemistry, 34: 1123-1127.

Carvalho, L.H.; Brandao, M.G.L.; Santos-Filho, D.; Lopes, J.L.F.; Krettli, A.U. 1992. A method for screening drugs against the liver stages of malaria using Plasmodium gallinaceum and Aedes mosquitos. Brazilian Journal of Medical and Biological Research, 25: 247-255.

Diniz, L.R.L.; Santana, P.C.; Ribeiro, A.P.A.F.; Portella, V.G.; Pacheco, L.F.; Meyer, N.B.; César, I.C.; Cosenza, G.P.; Brandão, M.G.L.; Vieira, M.A,R. 2009. Effect of triterpene saponins from roots of Ampelozizyphus amazonicus Ducke on diuresis in rats. Journal of Ethnopharmacology, 123: 275-279.

Ducke, J.A.; Martinez, R.V. 1994. Amazonian Ethnobotanical Dictionary. CRC Press, Boca Raton, USA. 215 pp.

Ellman, G. L.; Courtney, K. D.; Andress, V.; Feartherstone, R.M. 1961. A new and rapid colorimetric determination of acetylcholinesterase activity. Biochemical Pharmacology. 7: 88-95.

Francis, G.; Kerem, Z.; Makkar, H.P.S.; Becker, K. 2002. The biological action of saponins in animal systems: a review. British Journal of Nutrition, 88: 587-605.
Gauthier, C.; Legault, J.; Girard-Lalancette, K.; Mshvildadze, V.; Pichette, A. 2009. Haemolytic activity, cytotoxicity and membrane cell permeabilization of semi-synthetic and natural lupane- and oleanane-type saponins. Bioorganic \& Medicinal Chemistry, 17: 2002-2008.

Kato, E.T.M. 1996. Pharmacognostic study of the drug and the fluid extract of raspa-de-juá - Ziziphus joazeiro Martius. Lecta, 14: 9-28 (in Portuguese, with abstract in English).

Krettli, A.U.; Andrade-Neto, V.F.; Brandão, M.G.L.; Ferrari, W.M.S 2001. The Search for new antimalarial drugs from plants used to treat fever and malaria or plants ramdomly selected: a review. Memórias do Instituto Oswaldo Cruz. 96: 1033-1042.

Krettli, A.U.; Andrade-Neto, V.F. 2004. Search antimalarial drugs in the folk medicine. Ciência Hoje, 35: 70-73 (in Portuguese).

Lee, B.; Jung, K.; Kim, D-H. 2009. Timosaponin AIII, a saponin isolated from Anemarrhena asphodeloides, ameliorates learning and memory deficits in mice. Pharmacology, Biochemistry and Behavior, 93: 121-127.

Lima, R.B. 2006. Flora of the Ducke Reserve, Amazonas, Brazil: Rhamnaceae. Rodriguésia, 57: 247-249 (in Portuguese).

Matos, F.J.A. 2007. Medicinal Plants. 3 Ed. Universidade Federal do Ceará, Fortaleza, Ceará. 365p. il. (in Portuguese).

Mazza, M.; Pomponi, M.; Janiri, L.; Bria, P.; Mazza, S. 2007. Omega-3 fatty acids and antioxidants in neurological and psychiatric diseases: An overview. Progress in NeuroPsychopharmacology \& Biological Psychiatry, 37: 12-26.

MEB - Movimento de Educação de Base - Parintins-AM. 1993. Popular medicine recipes. Universidade Federal do Amazonas, Manaus, Amazonas. 26 pp. (in Portuguese).

Mishra, T.; Bhatia, A. 2010. Augmentation of expression of immunocytes' functions by seed extract of Ziziphus mauritiana (Lamk.). Journal of Ethnopharmacology, 127: 341-347.

Oliveira, D.R. 2004. Etnobotanical survey of medicinal plants used in the city of Oriximina' (Pará state) with etnopharmacology focus to the Lippia genus. Dissertação de Mestrado. Universidade Federal do Rio de Janeiro, Rio de Janeiro. 149 pp (in Portuguese).

Oliveira, D.R.; Leitão, GG.; Santos, S.S.; Bizzo, H.R.; Lopes, D.; Alviano, C.S.; Alviano, D.S.; Leitão, S.G. 2006. Ethnopharmacological study of two Lippia species from Oriximiná, Brazil. Journal of Ethnopharmacology, 108: 103-108.

Oliveira, D.R.; Leitão, G.G.; Castro, N.G.; Pinto, S.C.; Leitão, S.G. 2007. Screening of plant extracts on in vitro inhibition of acetylcholinesterase: a critical study. Jornal Brasileiro de Fitomedicina, 5: 6 (in Portuguese).

Oliveira, D.R. 2009. Bioprospecting of vegetable species of the traditional knowledge associated in quilombola communities from Oriximiná$P A$. Tese (Doutorado) - Núcleo de Pesquisas em Produtos Naturais, Universidade Federal do Rio de Janeiro, Rio de Janeiro. 303 pp. (in Portuguese).

Oliveira, D.R.; Leitão, S.G.; O’Dwyer, E.C.; Leitão, G.G.; ARQMO. 2010. Authorization of the traditional knowledge associated access for bioprospecting purposes: The case of UFRJ and the Association of the Oriximiná Quilombola Communities - 
ARQMO. Revista Fitos, 5: 59-76. (in Portuguese, with abstract in English).

OMS - Organização Mundial da Saúde. 1998. The international statistical classification of diseases and related health problems. Vol. 1. 10a Revisão. 6 Ed. EDUSP, São Paulo, Brasil. 1191 pp. (in Portuguese).

Panossian, A; Wikman, G; Wagner, H. 1999. Plant adaptogens II. Earlier and more recent aspects and concepts on their mode of action. Phytomedicine, 6: 287-300.

Revilla, J. 2002. Useful plants of the Amazon basin. Vol. 1. Instituto Nacional de Pesquisas da Amazônia / SEBRAE-AM, Manaus, Amazonas. 858 pp. (in Portuguese).

Rodrigues, R.M. 1989. The amazonian flora. CEJUP, Belém, Pará, 462 pp (in Portuguese).

Rodrigues, E. 2006. Plants and animals utilized as medicines in the Jaú National Park (JNP), Brazilian Amazon. Phytotherapy Research, 20: 378-391.

Rosas, L.V.; Cordeiro, M.S.C.; Nascimento, S.K.R.; Januário, A.H.; França, S.C.; Nomizo, A.; Toldo, M.P.A.; Albuquerque, S.; Pereira, P.S. 2007. In vitro evaluation of the cytotoxic and trypanocidal activities of Ampelozizyphus amazonicus (Rhamnaceae). Brazilian Journal of Medical and Biological Research, 40: 663-670.

Santos, A.M.S.; Kahwage, C.C.; Ferreira, M.R.C.; Sampaio, N.A. 2005. Traditional medicines in the Rio Negro Valley (Amazonas state, Brazil). Observations on the ethnopharmacology and the use of the plant saracuramirá (Ampelozizyphus amazonicus): Pharmacological activity and/or symbolic efficacy. Boletim do Museu Paraense Emílio Goeldi: Série Ciências Humanas, 1: $137-$ 147. (in Portuguese, with abstract in English).
Santos, F.S.D.; Muaze, M.A.F. 2002. Traditions in movement: An ethnohistory of the health and illness in the valleys of the Acre and Purus rivers. Paralelo 15, Brasília, Distrito Federal. 170 pp. (in Portuguese).

Scheltens, P.; Kamphuis, P.J.G.H.; Verhey, F.R.J.; Olde Rikkert, M.G.M.; Wurtman, R.J.; Wilkinson, D.; Twisk, J.W.R.; Kurz, A. 2010. Efficacy of a medical food in mild Alzheimer's disease: A randomized, controlled trial. Alzheimer's \& Dementia, 6: 1-10.

Silva, J.R.A.; Correa, G.M.; Carvalho, J.R.; Costa, R.A.; Pinheiro, M.L.B.; Araujo, L.M.; Amaral, A.C.F. 2009. Analyses of Ampelozizyphus amazonicus, a plant used in folk medicine of the Amazon Region. Pharmacognosy Magazine, 4: 75-80.

Sparg, S.G.; Light, M.E.; van Staden, J. 2004. Biological activities and distribution of plant saponins. Journal of Ethnopharmacology, 94: 219-243.

Thompson, E.C.; Juan, Z. 2006. Comparative cultural salience: Measures using free-list Data. Field Methods, 18: 398-411.

Vieira, L.S. 1992. Amazonian phytotherapy. Editora Agronômica Ceres, São Paulo, Brasil. 347 pp. (in Portuguese).

Yang, Z.-G.; Sun, H.-X.; Fang, W.-H. 2005. Haemolytic activities and adjuvant effect of Astragalus membranaceus saponins (AMS) on the immune responses to ovalbumin in mice. Vaccine, 23: 5196-5203.

WHO - World Health Organization. 1998. Determination of foaming index, p. 46. In: Quality control for medicinal plant materials. WHO/PHARM/92.559/Rev.1, Geneva.

Recebido em 07/06/2010

Aceito em 24/11/2010 